\title{
Study of introgression lines of common wheat Triticum aestivum/Triticum miguschovae for resistance to leaf rust
}

\author{
R.O. Davoyan*, I.V. Bebykina, E.R. Davoyan, D.S. Mikov, Y.S. Zubanova, D.M. Boldakov, A.N. Zinchenco
}

National Center of Grain named after P.P. Lukyanenko, Krasnodar, Russia

DOI 10.18699/ICG-PlantGen2019-17

(c) Autors, 2019

*e-mail: davoyanro@mail.ru

\begin{abstract}
The evaluation of resistance to leaf rust, the cytological and molecular analysis of 106 introgression lines of common wheat obtained on the basis of the synthetic form Triticum miguschovae Zhir were performed. Cytological stability of lines was established. 53 lines with high and 23 with medium resistance to leaf rust were selected. Two lines with the Lr39 gene and 26 lines with the Lr50 gene were identified with the use of molecular markers. Other resistant lines can presumably carry genes for resistance to leaf rust other than Lr39 or Lr50.

Key words: T. aestivum; T. miguschovae; introgression lines; resistance to leaf rust; cytological analysis; molecular markers.
\end{abstract}

\section{Introduction}

Leaf rust (Puccinia recondita Rob. ex Desm. f. sp. tritici Erikss.et Henn) is one of the most widespread and harmful diseases of wheat. The most effective way to control the infestation is by breeding resistant varieties. This work assumes the presence of a sufficient diversification of effective genes for resistance, including new ones. Of great interest are the species Triticum timopheevii Zhuk. (GGAA) and its natural mutant T. militinae Zhuk. et Migush., and Aegilops tauschii Coss. (DD) as sources of resistance to diseases (Friebe et al., 1996; McIntosh et al., 2005). The synthetic form T. miguschovae (GGA t $\left.A^{t} \mathrm{DD}\right)$ was used as a "genetic bridge' to transfer leaf rust resistance from these species to common wheat, where the D genome from Ae. tauschii was added to the AG genomes from T. militinae (Zhirov, 1980). To date, a large number of introgression lines and six varieties of winter common wheat have been obtained with the use of this form (Davoyan et al., 2012).

This article presents the results of the evaluation of 106 T. aestivum/T. miguschovae introgression lines of common wheat for resistance to leaf rust, cytological stability and the identification of previously transferred effective genes, Lr39 and $\operatorname{Lr} 50$.

\section{Materials and methods}

$106 \mathrm{BC}_{2} \mathrm{~F}_{22}-\mathrm{BC}_{4} \mathrm{~F}_{16}$ introgression lines of common wheat, obtained from crossing T. miguschovae with the varieties 'Bezostaya1', 'Kavkaz' and 'Skiphyanka' (MB, MK and $\mathrm{MKC}$ lines) susceptible to leaf rust were used as objects of research.

The cytological analysis was performed by studying chromosome paring in metaphase I of meiosis.

The reaction of plants to leaf rust infestation was evaluated using the international scale by Maines and Jackson in the field (Mains, Jackson, 1926). The plants with reaction types from 0 to 2 were classified as resistant. Plants with intermediate reaction types, between 0 and 1 , were designated as 01 . Plants with reaction types 3 and 4 were considered susceptible.

DNA of wheat was extracted from 5-7-day-old bleachedout sprouts using a method by Plaschke with the coauthors
(1995). Identification of genes was done with the use of molecular marker GDM-35 for Lr39 gene (Huang et al., 2001) and the GDM87 and WMS382 markers for the Lr50 gene (Brown-Guedira et al., 2003). Wheat lines KS86WGRC02 and KS96WGRC36 with genes for resistance to leaf rust, Lr39 and Lr50, respectively, were used as positive controls for identification of known genes.

\section{Results and discussion}

As the main aim of the work was to transfer resistance to diseases from T. miguschovae to common wheat, the lines were evaluated for resistance to one of the most widespread and harmful of them, leaf rust. The characteristics of the population of lines assessed for resistance to this pathogen in 20162018 is presented in Table 1 . The assessment of the lines revealed their difference in resistance to leaf rust in all combinations.

Highly resistant lines with reaction types 01 and 1 , medium resistant (reaction type 2), medium susceptible (reaction type 3 ) and highly susceptible (reaction type 4) lines were identified.

Fifty-three lines showed high resistance to leaf rust. 23 lines were medium resistant and 22 lines were susceptible to a local population of leaf rust. Also, 8 lines with a heterogeneous reaction type were revealed, where single pustules with treaction type 3 appeared on the background of high resistance (reaction type 1). Wide polymorphism of lines in resistance to leaf rust can indicate different introgressions of genetic material from T. miguschovae to the common wheat genome.

One of the basic conditions for the use of introgression lines as donors is their cytological stability, because it is closely connected with normal plant ontogenesis. Based on the fact that the lines analyzed were obtained by means of backcrosses from a large number of self-pollinating generations, it is very likely that they are meiotically stable. To acknowledge this, a cytological analysis of 20 resistant lines with reaction types 01,1 and 2 was performed. For the establishment of cytological stability, chromosome pairing in metaphase I of meiosis in 3-4 plants from every line was studied. The number of the cells studied in each line varied from 100 to 140 . The lines 
Table 1

Characteristics of the population of T. aestivum/T. miguschovae introgression lines for resistance to leaf rust in 2016-2018

\begin{tabular}{llllll}
\hline Cross combination & \multicolumn{5}{c}{ Number of lines with reaction type to leaf rust } \\
& 01 & 1 & 2 & $3-4$ & $1 / 3$ \\
\hline MB & 5 & 6 & 6 & 4 & 3 \\
MK & 15 & 11 & 12 & 6 & 2 \\
MKS & 10 & 6 & 5 & 12 & 3 \\
Total & 30 & 23 & 23 & 22 & 8 \\
\hline
\end{tabular}
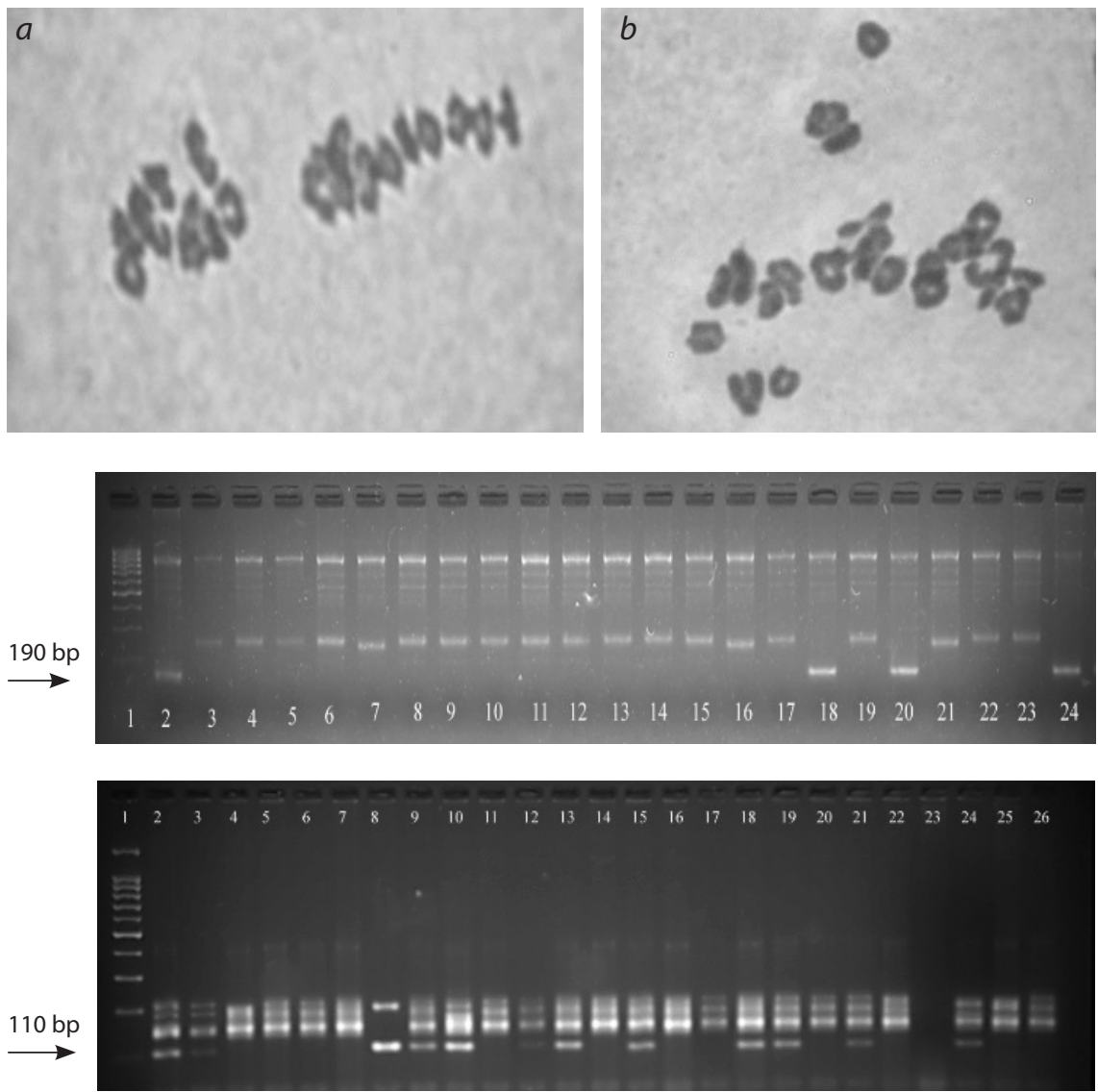

Figure 1. Association of chromosomes in metaphase I of meiosis in lines MK509-21" (a) and MB1801-21" (b)

Figure 2. Products of amplification with the use of the GDM35-R and GDM35-L primer pairs to the diagnostic marker linked to $L r 39$, a gene for resistance to leaf rust. 1, marker of length; $2, T C L r 39$ 3, variety 'Avrora'; 4-23, lines; 24, T. miguschovae; 18, line MK452; 20, line MKC985.

Figure 3. Products of amplification with the use of the GDM87-L/R primer pair to the diagnostic marker linked to the leaf rust resistance gene Lr50. 1, marker of length; 2, T. miguschovae; 3, KS96WGRC36 (Lr50); 4, variety 'Bezostaya 1 ; $5-26$, the lines obtained on the basis of T. miguschovae. were cytologically stable where they had more than $90 \%$ of cells with the bivalent $\left(21^{\mathrm{II}}\right)$ configuration of chromosomes (Figure 1).

All lines analyzed formed the $21^{\mathrm{II}}$ bivalent and were cytologically stable regardless of their origin and degree of resistance. The efficiency of selection for resistance to diseases depends on the presence of information about identification of genes. The genes the resistance to leaf rust $\operatorname{Lr} 18$ and $\operatorname{Lr} 50$ were transferred from $T$. timopheevii to common wheat, and Lr21, Lr32, Lr39, Lr41, Lr42, Lr43 were transferred from Ae. tauschii (McIntosh, 2005). It is possible that T. miguschovae and the lines obtained on its basis carry these genes. Of these resistance genes, $\operatorname{Lr} 39$ and $L r 41$ are effective and $L r 50$ is partially effective in various regions of Russia nowadays (Zhemchuzina, Kurkova, 2010). At the same time, the identity of genes $L r 39$ and $L r 41$ was established (Singh, 2004). In this connection, a screening of 76 resistance lines with genetic material from $T$. miguschovae for the presence of molecular markers linked to the $\operatorname{Lr} 39$ and $\operatorname{Lr} 50$ genes was performed. For identification of $L r 39$, a gene for resistance to leaf rust, the microsatellite marker GDM35 was used. GDM35 is widely used for screening varieties and lines in the USA (Sun et al., 2009). Specific fragments of amplification $190 \mathrm{bp}$ to the diagnostic marker GDM35, linked to the $L r 39$ gene were detected in the positive control line KS86WGRC02, the synthetic form T. miguschovae, and two highly resistant lines, MK452 and MKC985 (Figure 2).

Identification of the leaf rust resistance gene $L r 50$ was done with two microsatellite markers, $\mathrm{Xgwm} 382$ and $\mathrm{Xgdm} 87$, which outflank the $L r 50$ locus at a distance of 6.7 and $9.4 \mathrm{cM}$, respectively. The 110-bp fragment was determined after amplification with the use of the GDM87-L/R primers, and the 139-bp fragment appeared after amplification with the use of the $W M S 382-L / R$ primers. The presence of the $L r 50$ gene in the diagnosed sample was established upon revealing fragments of amplification of both markers. 
The $110 \mathrm{bp}$ fragment of amplification using the diagnostic marker GDM87 linked to the resistance gene $\operatorname{Lr} 50$ was identified in the positive control KS96WGRC36, the form T. miguschovae and in 32 resistance lines. The products of amplification of 22 lines are presented in Figure 3. The fragment specific to a marker linked to the $\operatorname{Lr} 50$ gene was revealed in lines MK295, MK425, MK538, MKS627, MB1681, MK1795, MB1885, MB2305, MK2497, and MB2519 (Nos. 8, $9,10,12,13,15,18,19,21$, and 24 , respectively).

\section{Conclusions}

The results obtained indicate the value of the studied lines for breeding for resistance to leaf rust. They are meiotically stable, different in resistance and can probably carry genes for resistance to leaf rust other than the effective genes $\operatorname{Lr} 39$ and Lr50. Genetic material from T. miguschovae was identified in introgression lines with the use of C-banding and FISH methods.

\section{References}

Brown-Guedira G., Singh S., Fritz A.K. Performance and mapping of leaf rust transferred to wheat from Triticum timopheevii subsp. armeniacum. Phytopathol. 2003;93:784-789.

Davoyan R.O., Bebyakina I.V., Davoyan O.R., Zinchenko A.N., Davoyan E.R., Kravchenko A.M., Zubanova Y.S. The use of synthe- tic forms in preservation and exploitation of the gene pool of wild common wheat relatives. Russian Journal of Genetics: Applied Research. 2012;2(6):480-485.

Friebe B., Jiang J., Raupp W.J. Characterization of wheat-alien translocations conferring resistance to diseases and pests: current status. Euphytica. 1996; 91:59-87.

Huang L. An RGA-like marker detects all known $L r 21$ leaf rust-resistance gene family members in Aegilops tauschii and wheat. Theor. Appl. Genet.2001;103(6-7):1007-1013.

Mains E.B. Fhysiologic specialization in leaf rust of wheat, Puccinia triticiana Erikss. Phytopatol. 1926;16:89-120.

McIntosh R.A. Catalogue of gene symbols for wheat: 2005 supplement. Ann. Wheat Newslet. 2005;51:272-285.

Plaschke J., Ganal M.W., Röder M.S. Detection of genetic diversity in closely related bread wheat using microsatellite markers. Theor. Appl. Genet. 1995;91:1001-1007.

Singh S. Lr41, Lr39, and a leaf rust resistance gene from Aegilops cylindrical may be allelic and are located on wheat chromosome 2DS. Theor. Appl. Genet. 2004;108:586-591.

Sun X., Bai G., Carver B.F. Molecular markers for wheat leaf rust resistance gene Lr41. Mol. Breedingm. 2009;23:311-321.

Zhemchuzina A., Kurkova N. Structure of population of Puccinia triticina in various regions of Russia in 2006-2008. Proc. of the BGRI Technical Workshop. St. Petersburg, Russia. May 30-31, 2010:27.

Zhirov E.G. Synthesis of a new hexaploid wheat. Trudi po prikladnoi botanike, genetike, selektsii. 1980;68:14-16 (in Russian).

Conflict of interest. The authors declare no conflict of interest. 